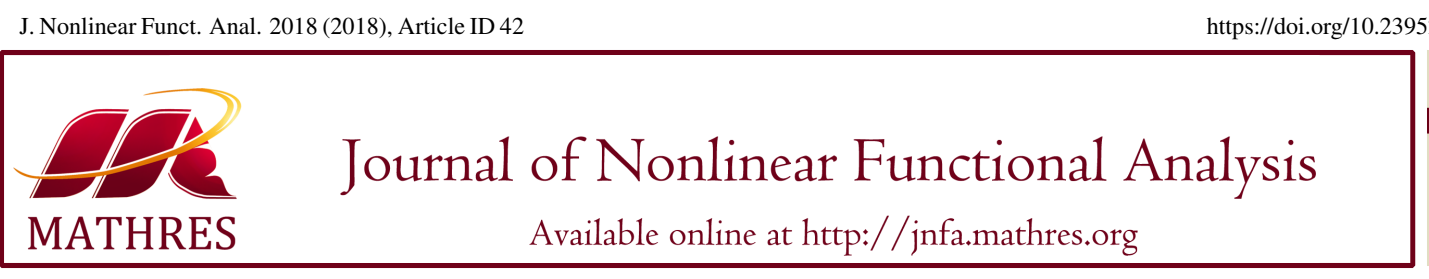

https://doi.org/10.23952/jnfa.2018.42

\title{
A HYBRID DESCENT ITERATIVE ALGORITHM FOR A SPLIT INCLUSION PROBLEM
}

\author{
YANTAO YANG ${ }^{1}$, QING YUAN ${ }^{2, *}$ \\ ${ }^{1}$ College of Mathematics and Computer Science, Yanan University, Yanan 716000, China \\ ${ }^{2}$ School of Mathematics and Statistics, Linyi University, Linyi 276000, China
}

\begin{abstract}
The purpose of this paper is to introduce a hybrid descent iterative algorithm for solving a split variational inclusion problem and a fixed point problem of a strict pseudocontraction mapping. We establish a strong convergence theorem of common solutions of the two problems in the framework of Hilbert spaces without any compact assumptions on any mapping.
\end{abstract} Keywords. Iterative algorithm; Monotone operator; Split inclusion problem; Variational inequality.

2010 Mathematics Subject Classification. 47H05, 47J20, 65K15.

\section{INTRODUCTION}

Throughout the paper unless otherwise stated, let $H_{1}$ and $H_{2}$ be two real Hilbert spaces endowed with inner products and induced norms denoted by $\langle\cdot, \cdot\rangle$ and $\|\cdot\|$, respectively, while $H$ refers to as any of these spaces. Let $D$ be a nonempty closed and convex subset of $H$ and Let $\operatorname{Proj}_{D}^{H}$ be the metric projection onto $D$. A very common problem in diverse areas of mathematics and physical science consists of trying to find a "solution" satisfying certain "constraints". This problem is referred to as a convex feasibility problem, which is to find a common element in a family of nonempty closed and convex subsets of a Hilbert space.

Let $C$ be a nonempty closed and convex subset of $H_{1}$ and let $Q$ be a nonempty closed and convex subset of $\mathrm{H}_{2}$. In 1994, Censor and Elfving [1] first introduced the following split feasibility problem for modelling inverse problems formulated as follows:

$$
\text { Find } x^{*} \in C \text { such that } A x^{*} \in Q \text {, }
$$

where $A: H_{1} \rightarrow H_{2}$ is a bounded linear mapping. This problem can also be viewed as a convex feasibility problem. There are a number of significant applications of the split feasibility problem in intensitymodulated radiation therapy, signal processing, image reconstruction and so on. An efficient algorithm

${ }^{*}$ Corresponding author.

E-mail addresses: yadxyyt@163.com (Y. Yang), yuanqing@lyu.edu.cn (Q. Yuan)

Received June 20, 2018; Accepted December 3, 2018.

(C)2018 Journal of Nonlinear Functional Analysis 
for solving (1.1) is the Byrne's $C Q$ algorithm. For any $x_{0} \in H_{1}$, the $C Q$ algorithm generates an iterative sequence as

$$
x_{n+1}=\operatorname{Proj}_{C}^{H_{1}}\left(I+\gamma A^{*}\left(\operatorname{Proj}_{Q}^{H_{2}}-I\right) A\right) x_{n},
$$

where $0<\gamma<2 /\|A\|^{2}$. It is known that the $C Q$ algorithm converges weakly to a solution of problem (1.1) if such a solution exists. To guarantee the strong convergence of the sequence, a number of regularization methods have been investigated to solve the split feasibility problem and their related convex optimization problems recently; see [2]-[10] and the references therein.

Let $M$ and $N$ be two maximal monotone operators on $H_{1}$ and $H_{2}$, respectively. In this paper, we study the following split variational inclusion problem: Find $x^{*} \in H_{1}$ such that

$$
0 \in M\left(x^{*}\right)
$$

and such that

$$
y^{*}=A x^{*} \in H_{2} \quad \text { solves } \quad 0 \in N\left(y^{*}\right) .
$$

In this paper, we use $\operatorname{SFP}(M, N)$ to denote the solution set of the split variational inclusion problem. Recently, the above split variational inclusion problem has been introduced and studied based on Mannlike methods by many authors; see $[11,12,13,14,15]$ and the references therein. In this paper, we investigate the split variational inclusion problem via a hybrid descent iterative algorithm in Hilbert spaces. Strong convergence theorems are established without any compact assumptions on mappings. The main results presented in this paper partly improve the results in $[6,8,11,12]$. The organization of this paper is as follows. In Section 2, we provide the necessary mathematical preliminaries. The last section is devoted to the strong convergence analysis of the hybrid descent iterative algorithm. Some subresults are derived as corollaries of the main results.

\section{Preliminaries}

Let $T$ be a mapping on $D$. The fixed-point set of $T$ is denoted by $\operatorname{Fix}(T)$. Recall that $T$ is said to be nonexpansive iff

$$
\|T x-T y\| \leq\|x-y\|, \quad \forall x, y \in D .
$$

$T$ is said to be quasi-nonexpansive iff $\operatorname{Fix}(T) \neq \emptyset$ and

$$
\|x-T y\| \leq\|x-y\|, \quad \forall x \in \operatorname{Fix}(T), y \in D .
$$

It is known that every nonexpansive mapping satisfies the following properties

$$
\langle T x-T y,(y-T y)-(x-T x)\rangle \leq \frac{1}{2}\|(x-T x)-(y-T y)\|^{2}, \quad \forall x, y \in D .
$$

In particular, every quasi-nonexpansive mapping satisfies the following properties

$$
\langle x-T y,(y-T y)\rangle \leq \frac{1}{2}\|y-T y\|^{2}, \quad \forall x \in F i x(T), y \in D .
$$

Recall that $T$ is said to be firmly nonexpansive iff

$$
\|T x-T y\|^{2} \leq\langle T x-T y, x-y\rangle, \quad \forall x, y \in D .
$$

Recall that $T$ is said to be $\kappa$-strictly pseudocontractive iff there is a real number $\kappa \in[0,1)$ such that

$$
\|T x-T y\|^{2} \leq\|x-y\|+\kappa\|(I-T) x-(I-T)\|^{2}, \quad \forall x, y \in D .
$$


The class of $\kappa$-strictly pseudocontractive mappings was first introduced and studied by Browder and Petryshy [16] in the framework of Hilbert spaces. Since then, many authors have studied fixed points of $\kappa$-strictly pseudocontractive mappings via different methods and techniques; see $[17,18,19,20,21]$ and the references therein.

Recall that a mapping $M: H \rightarrow 2^{H}$ is said to be monotone iff, for all $x, y \in H_{1}, u \in M x$ and $v \in M y$

$$
\langle x-y, u-v\rangle \geq 0 .
$$

It is said to be maximal iff the $\operatorname{Graph}(M)$ is not properly contained in the graph of any other monotone mapping. It is known that a monotone mapping $M$ is maximal iff for $(x, u) \in H \times H,\langle x-y, u-v\rangle \geq 0$, for every $(y, v) \in \operatorname{Graph}(M)$ implies that $u \in M(x)$. The resolvent mapping, $J_{r}^{M}: H \rightarrow H$ associated with $M$, is defined by

$$
J_{r}^{M} x=(I+r M)^{-1}(x), \forall x \in H,
$$

where $r>0$ is some real number and $I$ stands for identity operator on $H$. It is known that $J_{r}^{M}$ is singlevalued, nonexpansive and firmly nonexpansive. The resolve operator palys an important role in many convex optimization problem; see $[3,8,9,13,15]$ and the references therein.

Lemma 2.1. [16] Let D be a nonempty convex and closed subset of a Hilbert space $H$. Let $T$ be a $\kappa$-strict pseudocontraction and let $\left\{\beta_{n}\right\}$ be a sequence in $(0,1)$. Define a mapping $S$ by $S x=\left(1-\beta_{n}\right) T x+\beta_{n} x$, $\forall x \in D$. If $\beta \in[\kappa, 1)$, then $S$ is nonexpansive and $\operatorname{Fix}(S)=F i x(T)$.

Lemma 2.2. [22] Let $H$ be a real Hilbert space and let $M$ be a maximal operator. For $\lambda>0$ and $\mu>0$ we have

$$
(I+\mu A)^{-1}\left(\frac{\mu}{\lambda} x+\left(1-\frac{\mu}{\lambda}\right)(I+\lambda M)^{-1} x\right)=(I+\lambda M)^{-1} x, \forall x \in H .
$$

Lemma 2.3. [23] Let $\left\{\alpha_{n}\right\}$ and $\left\{\beta_{n}\right\}$ be sequences of real numbers such that $\alpha_{n} \in[0,1] \sum_{n=1}^{\infty} \alpha_{n}=\infty$ and $\limsup _{n \rightarrow \infty} \beta_{n} \leq 0$. Let $\left\{\lambda_{n}\right\}$ be a sequence of nonnegative real numbers such that $\lambda_{n+1} \leq \alpha_{n} \beta_{n}+$ $\left(1-\alpha_{n}\right) \lambda_{n}$. Then $\lim _{n \rightarrow \infty} \lambda_{n}=0$.

Lemma 2.4. [24] Let $H$ be a Hilbert space and let $F$ is a $\eta$-strongly monotone, $\mathscr{L}$-Lipschitz continuous mapping on $H$. Define a mapping $T^{\alpha}: H \rightarrow H$ by $T^{\alpha} x=(I-\mu \alpha F) x, \forall x \in H_{1}$, where $\alpha$ is a real number in $(0,1)$. If $0<\mu \in\left(0, \frac{2 \eta}{\mathscr{L}^{2}}\right)$, then $T^{\alpha}$ is a contraction, that is, $\left\|T^{\alpha} x-T^{\alpha} y\right\| \leq(1-\alpha \tau)\|x-y\|, \forall x, y \in H$, where $\tau=1-\sqrt{1-\mu\left(2 \eta-\mu \mathscr{L}^{2}\right)} \in(0,1]$.

Lemma 2.5. [16] Let D be a nonempty convex and closed subset of a Hilbert space H. Let T be a $\kappa$-strict pseudocontraction with fixed points. Then I-T, where I is the identity mapping, is demiclosed on any point in $D$.

\section{MAIN RESULTS}

Theorem 3.1. Let $C$ be a nonempty closed and convex subset of a Hilbert space $H_{1}$ and let $Q$ be a nonempty closed and convex subset of a real Hilbert space $\mathrm{H}_{2}$. Let $\mathrm{T}$ be a $\mathrm{\kappa}$-strictly pseudocontractive mapping on $H_{1}$ with fixed points. Let $A: H_{1} \rightarrow H_{2}$ be a bounded linear operator and let $A^{*}$ be the adjoint operator of $A$. Let $F: H_{1} \rightarrow H_{1}$ be a $\mathscr{L}$-Lipschitz continuous and $\tau$-strongly monotone mapping. Let $M$ be a maximal monotone mapping on $\mathrm{H}_{1}$ and let $\mathrm{N}$ be a maximal monotone mapping on $\mathrm{H}_{2}$. Assume that 
$\operatorname{Fix}(T) \cap \operatorname{SFP}(M, N) \neq \emptyset$. Let $\gamma$ be a positive real number. Let $\left\{\alpha_{n}\right\}$ and $\left\{\beta_{n}\right\}$ be sequences in $(0,1)$ and let $\left\{r_{n}\right\}$ be a positive sequence. Let $\left\{x_{n}\right\}$ be a sequence generated by the following process: $x_{1} \in H_{1}$ and

$$
\left\{\begin{array}{l}
x_{n+1}=T_{\beta_{n}} y_{n}-\mu \alpha_{n} F T_{\beta_{n}} y_{n}, \\
y_{n}=J_{r_{n}}^{M}\left(x_{n}+\gamma A^{*}\left(J_{r_{n}}^{N}-I\right) A x_{n}\right), \quad n \geq 1,
\end{array}\right.
$$

where $T_{\beta_{n}}=\left(1-\beta_{n}\right) T+\beta_{n} I$. Assume that $\lim _{n \rightarrow \infty} \alpha_{n}=0, \sum_{n=1}^{\infty} \alpha_{n}=\infty$ and $\sum_{n=1}^{\infty}\left|\alpha_{n+1}-\alpha_{n}\right|<\infty$, $\gamma \in\left(0, \frac{1}{\|A\|^{2}}\right), \mu$ is a real number in $\left(0, \frac{2 \tau}{\mathscr{L}^{2}}\right),\left\{r_{n}\right\}$ is a real number sequence such that $\liminf _{n \rightarrow \infty} r_{n}>0$, $\sum_{n=1}^{\infty}\left|r_{n+1}-r_{n}\right|<\infty,\left\{\beta_{n}\right\}$ is a real number sequence in $[\kappa, 1)$ such that $\sum_{n=1}^{\infty}\left|\beta_{n+1}-\beta_{n}\right|<\infty$. Then $\left\{x_{n}\right\}$ converge strongly to $\bar{x} \in \operatorname{SFP}(M, N) \cap F i x(T)$, which is the unique solution of the following variational inequality

$$
\langle F \bar{x}, \bar{x}-y\rangle \leq 0, \quad \forall y \in \operatorname{SFP}(M, N) \cap F i x(T) .
$$

Proof. Since the set of common solutions is nonempty, we can fix a point in $\operatorname{SFP}(M, N) \cap \operatorname{Fix}(T)$, say, $x$. It follows that $A x=J_{r_{n}}^{N} A x, x=J_{r_{n}}^{M} x$ and $x=T x$. Note that

$$
\begin{aligned}
\left\|y_{n}-x\right\|^{2}= & \left\|J_{r_{n}}^{M} x-J_{r_{n}}^{M}\left(x_{n}+\gamma A^{*}\left(J_{r_{n}}^{N}-I\right) A x_{n}\right)\right\|^{2} \\
\leq & \left\|x-x_{n}-\gamma A^{*}\left(J_{r_{n}}^{N}-I\right) A x_{n}\right\|^{2} \\
\leq & \left\|x-x_{n}\right\|^{2}+2 \gamma\left\langle A^{*}\left(J_{r_{n}}^{N}-I\right) A x_{n}, x_{n}-x\right\rangle+\gamma^{2}\|A\|^{2}\left\|\left(J_{r_{n}}^{N}-I\right) A x_{n}\right\|^{2} \\
\leq & \left\|x-x_{n}\right\|^{2}+2 \gamma\left\langle A\left(x_{n}-x\right)+\left(J_{r_{n}}^{N}-I\right) A x_{n},\left(J_{r_{n}}^{N}-I\right) A x_{n}\right\rangle \\
& -2 \gamma\left\|\left(J_{r_{n}}^{N}-I\right) A x_{n}\right\|^{2}+\gamma^{2}\|A\|^{2}\left\|\left(J_{r_{n}}^{N}-I\right) A x_{n}\right\|^{2} .
\end{aligned}
$$

Since both $J_{r_{n}}^{N}$ and $J_{r_{n}}^{M}$ are nonexpansive, we find from (2.1) that

$$
2 \gamma\left\langle A\left(x_{n}-x\right)+\left(J_{r_{n}}^{N}-I\right) A x_{n},\left(J_{r_{n}}^{N}-I\right) A x_{n}\right\rangle \leq \gamma\left\|\left(J_{r_{n}}^{N}-I\right) A x_{n}\right\|^{2}
$$

Hence, one has

$$
\left\|y_{n}-x\right\|^{2} \leq \gamma\left(\gamma\|A\|^{2}-1\right)\left\|\left(J_{r_{n}}^{N}-I\right) A x_{n}\right\|^{2}+\left\|x-x_{n}\right\|^{2} .
$$

From the restriction on constant $\gamma$, one reaches that $\left\|y_{n}-x\right\| \leq\left\|x-x_{n}\right\|$. Using Lemma 2.1, one finds that $T_{\beta_{n}}$ is nonexpansvie and $\operatorname{Fix}(T)=\operatorname{Fix}\left(T_{\beta_{n}}\right)$ for each $n \geq 1$. So,

$$
\left\|T_{\beta_{n}} y_{n}-x\right\| \leq\left\|y_{n}-x\right\| \leq\left\|x-x_{n}\right\| .
$$

This in turn implies that

$$
\begin{aligned}
\left\|x_{n+1}-x\right\| & \leq \mu \alpha_{n}\|F x\|+\left\|\left(I-\mu \alpha_{n} F\right) T_{\beta_{n}} y_{n}-\left(I-\mu \alpha_{n} F\right) x\right\| \\
& \leq \mu \alpha_{n}\|F x\|+\left(1-\tau \alpha_{n}\right)\left\|T_{\beta_{n}} y_{n}-x\right\| \\
& \leq \mu \alpha_{n}\|F x\|+\left(1-\tau \alpha_{n}\right)\left\|x_{n}-x\right\| \\
& \leq\left(1-\tau \alpha_{n}\right)\left\|x_{n}-x\right\|+\tau \alpha_{n} \frac{\mu\|F x\|}{\tau} \\
& \leq \max \left\{\frac{\mu\|F x\|}{\tau},\left\|x_{n}-x\right\|\right\} \\
& \leq \ldots \\
& \leq \max \left\{\frac{\mu\|F x\|}{\tau},\left\|x_{1}-x\right\|\right\} .
\end{aligned}
$$


This proves that $\left\{x_{n}\right\}$ is bounded. It is easy to see that $\left\{y_{n}\right\}$ is also bounded. Note that

$$
\begin{aligned}
\left\|y_{n}-y_{n+1}\right\| \leq & \left\|J_{r_{n}}^{M}\left(x_{n}+\gamma A^{*}\left(J_{r_{n}}^{N}-I\right) A x_{n}\right)-J_{r_{n+1}}^{M}\left(x_{n+1}+\gamma A^{*}\left(J_{r_{n+1}}^{N}-I\right) A x_{n+1}\right)\right\| \\
\leq & \left\|J_{r_{n}}^{M}\left(x_{n}+\gamma A^{*}\left(J_{r_{n}}^{N}-I\right) A x_{n}\right)-J_{r_{n}}^{M}\left(x_{n+1}+\gamma A^{*}\left(J_{r_{n+1}}^{N}-I\right) A x_{n+1}\right)\right\| \\
& +\left\|J_{r_{n}}^{M}\left(x_{n+1}+\gamma A^{*}\left(J_{r_{n+1}}^{N}-I\right) A x_{n+1}\right)-J_{r_{n+1}}^{M}\left(x_{n+1}+\gamma A^{*}\left(J_{r_{n+1}}^{N}-I\right) A x_{n+1}\right)\right\| \\
\leq & \left\|\left(x_{n}+\gamma A^{*}\left(J_{r_{n}}^{N}-I\right) A x_{n}\right)-\left(x_{n+1}+\gamma A^{*}\left(J_{r_{n+1}}^{N}-I\right) A x_{n+1}\right)\right\| \\
& +\left\|J_{r_{n}}^{M}\left(x_{n+1}+\gamma A^{*}\left(J_{r_{n+1}}^{N}-I\right) A x_{n+1}\right)-J_{r_{n+1}}^{M}\left(x_{n+1}+\gamma A^{*}\left(J_{r_{n+1}}^{N}-I\right) A x_{n+1}\right)\right\| .
\end{aligned}
$$

Using Lemma 2.2, we see that

$$
\begin{aligned}
\| & J_{r_{n}}^{M}\left(x_{n+1}+\gamma A^{*}\left(J_{r_{n+1}}^{N}-I\right) A x_{n+1}\right)-J_{r_{n+1}}^{M}\left(x_{n+1}+\gamma A^{*}\left(J_{r_{n+1}}^{N}-I\right) A x_{n+1}\right) \| \\
= & \| J_{r_{n}}^{M}\left(\frac{r_{n}}{r_{n+1}}\left(x_{n+1}+\gamma A^{*}\left(J_{r_{n+1}}^{N}-I\right) A x_{n+1}\right)+\left(1-\frac{r_{n}}{r_{n+1}}\right) J_{r_{n+1}}^{M}\left(x_{n+1}+\gamma A^{*}\left(J_{r_{n+1}}^{N}-I\right) A x_{n+1}\right)\right) \\
& -J_{r_{n}}^{M}\left(x_{n+1}+\gamma A^{*}\left(J_{r_{n+1}}^{N}-I\right) A x_{n+1}\right) \| \\
\leq & \|\left(\frac{r_{n}}{r_{n+1}}\left(x_{n+1}+\gamma A^{*}\left(J_{r_{n+1}}^{N}-I\right) A x_{n+1}\right)+\left(1-\frac{r_{n}}{r_{n+1}}\right) J_{r_{n+1}}^{M}\left(x_{n+1}+\gamma A^{*}\left(J_{r_{n+1}}^{N}-I\right) A x_{n+1}\right)\right) \\
& -\left(x_{n+1}+\gamma A^{*}\left(J_{r_{n+1}}^{N}-I\right) A x_{n+1}\right) \| \\
\leq & \left.\frac{\left|r_{n+1}-r_{n}\right|}{r_{n+1}} \| J_{r_{n+1}}^{M}\left(x_{n+1}+\gamma A^{*}\left(J_{r_{n+1}}^{N}-I\right) A x_{n+1}\right)\right)-\left(x_{n+1}+\gamma A^{*}\left(J_{r_{n+1}}^{N}-I\right) A x_{n+1}\right) \| .
\end{aligned}
$$

Using Lemma 2.2 again, we also have

$$
\begin{aligned}
& \left\|\left(x_{n}+\gamma A^{*}\left(J_{r_{n}}^{N}-I\right) A x_{n}\right)-\left(x_{n+1}+\gamma A^{*}\left(J_{r_{n+1}}^{N}-I\right) A x_{n+1}\right)\right\| \\
& =\left\|x_{n}-x_{n+1}+\gamma A^{*}\left(\left(J_{r_{n}}^{N}-I\right) A x_{n}-\left(J_{r_{n+1}}^{N}-I\right) A x_{n+1}\right)\right\| \\
& \leq\left\|x_{n}-x_{n+1}+\gamma A^{*}\left(A x_{n+1}-A x_{n}\right)\right\|+\left\|\gamma A^{*}\left(J_{r_{n}}^{N} A x_{n}-J_{r_{n+1}}^{N} A x_{n+1}\right)\right\| \\
& \leq\left(1-\gamma\|A\|^{2}\right)\left\|x_{n}-x_{n+1}\right\|+\gamma\|A\|\left\|J_{r_{n}}^{N} A x_{n}-J_{r_{n}}^{N}\left(\frac{r_{n}}{r_{n+1}} A x_{n+1}+\left(1-\frac{r_{n}}{r_{n+1}}\right) J_{r_{n+1}}^{N} A x_{n+1}\right)\right\| \\
& \leq\left(1-\gamma\|A\|^{2}\right)\left\|x_{n}-x_{n+1}\right\|+\gamma\|A\|\left(\frac{r_{n+1}-r_{n} \mid}{r_{n+1}}\left\|J_{r_{n+1}}^{N}\left(A x_{n+1}-A x_{n+1}\right)\right\|+\left\|A x_{n+1}-A x_{n}\right\|\right) \\
& \leq\left\|x_{n}-x_{n+1}\right\|+\frac{\gamma\|A\|\left|r_{n+1}-r_{n}\right|}{r_{n+1}}\left\|J_{r_{n+1}}^{N}\left(A x_{n+1}-A x_{n+1}\right)\right\| .
\end{aligned}
$$

From (3.3), (3.4) and (3.5), we see that

$$
\begin{aligned}
& \left\|y_{n}-y_{n+1}\right\| \\
& \leq\left\|x_{n}-x_{n+1}\right\|+\frac{\gamma\|A\|\left|r_{n+1}-r_{n}\right|}{r_{n+1}}\left\|J_{r_{n+1}}^{N}\left(A x_{n+1}-A x_{n+1}\right)\right\| \\
& \left.\quad+\frac{\left|r_{n+1}-r_{n}\right|}{r_{n+1}} \| J_{r_{n+1}}^{M}\left(x_{n+1}+\gamma A^{*}\left(J_{r_{n+1}}^{N}-I\right) A x_{n+1}\right)\right)-\left(x_{n+1}+\gamma A^{*}\left(J_{r_{n+1}}^{N}-I\right) A x_{n+1}\right) \| .
\end{aligned}
$$


Hence, one has

$$
\begin{aligned}
& \left\|T_{\beta_{n}} y_{n}-T_{\beta_{n+1}} y_{n+1}\right\| \\
& \leq\left\|T_{\beta_{n}} y_{n}-T_{\beta_{n}} y_{n+1}\right\|+\left\|T_{\beta_{n}} y_{n+1}-T_{\beta_{n+1}} y_{n+1}\right\| \\
& \leq\left\|y_{n}-y_{n+1}\right\|+\left\|T_{\beta_{n}} y_{n+1}-T_{\beta_{n+1}} y_{n+1}\right\| \\
& \leq \\
& \leq y_{n}-y_{n+1}\left\|+\left|\beta_{n+1}-\beta_{n}\right|\right\| T y_{n+1}-y_{n+1} \| \\
& \leq\left\|x_{n}-x_{n+1}\right\|+\frac{\gamma\|A\|\left|r_{n+1}-r_{n}\right|}{r_{n+1}}\left\|J_{r_{n+1}}^{N}\left(A x_{n+1}-A x_{n+1}\right)\right\| \\
& \left.\quad+\frac{\left|r_{n+1}-r_{n}\right|}{r_{n+1}} \| J_{r_{n+1}}^{M}\left(x_{n+1}+\gamma A^{*}\left(J_{r_{n+1}}^{N}-I\right) A x_{n+1}\right)\right)-\left(x_{n+1}+\gamma A^{*}\left(J_{r_{n+1}}^{N}-I\right) A x_{n+1}\right) \| \\
& \quad+\left|\beta_{n+1}-\beta_{n}\right|\left\|T y_{n+1}-y_{n+1}\right\| .
\end{aligned}
$$

On the other hand, one has

$$
\begin{aligned}
\left\|x_{n+2}-x_{n+1}\right\| \leq & \left\|\left(I-\mu \alpha_{n+1} F\right) T_{\beta_{n+1}} y_{n+1}-\left(I-\mu \alpha_{n+1} F\right) T_{\beta_{n}} y_{n}\right\| \\
& +\left\|\left(I-\mu \alpha_{n+1} F\right) T_{\beta_{n}} y_{n}-\left(I-\mu \alpha_{n} F\right) T_{\beta_{n}} y_{n}\right\| \\
\leq & \left(1-\tau \alpha_{n+1}\right)\left\|T_{\beta_{n+1}} y_{n+1}-T_{\beta_{n}} y_{n}\right\|+\mu\left|\alpha_{n+1}-\alpha_{n}\right|\left\|F T_{\beta_{n}} y_{n}\right\| .
\end{aligned}
$$

Combing (3.7) with (3.8), one arrives at

$$
\begin{aligned}
\left\|x_{n+2}-x_{n+1}\right\| \leq & \mu\left|\alpha_{n+1}-\alpha_{n}\right|\left\|F T_{\beta_{n}} y_{n}\right\|+\left(1-\tau \alpha_{n+1}\right)\left\|x_{n}-x_{n+1}\right\| \\
& +\frac{\gamma\|A\||| r_{n+1}-r_{n} \mid}{r_{n+1}}\left\|J_{r_{n+1}}^{N}\left(A x_{n+1}-A x_{n+1}\right)\right\| \\
& \left.+\frac{\left|r_{n+1}-r_{n}\right|}{r_{n+1}} \| J_{r_{n+1}}^{M}\left(x_{n+1}+\gamma A^{*}\left(J_{r_{n+1}}^{N}-I\right) A x_{n+1}\right)\right)-\left(x_{n+1}+\gamma A^{*}\left(J_{r_{n+1}}^{N}-I\right) A x_{n+1}\right) \| \\
& +\left|\beta_{n+1}-\beta_{n}\right|\left\|T y_{n+1}-y_{n+1}\right\| .
\end{aligned}
$$

An application of Lemma 2.3 to (3.9) yields that

$$
\lim _{n \rightarrow \infty}\left\|x_{n+1}-x_{n}\right\|=0 .
$$

Since $J_{r_{n}}^{N}$ is firmly nonexpansive, we obtain from (2.1) that

$$
\begin{aligned}
&\left\|y_{n}-x\right\|^{2} \\
& \leq\left\langle y_{n}-x, x_{n}+\gamma A^{*}\left(J_{r_{n}}^{N}-I\right) A x_{n}-x\right\rangle \\
&= \frac{1}{2}\left\{\left\|y_{n}-x\right\|^{2}-\left\|x_{n}+\gamma A^{*}\left(J_{r_{n}}^{N}-I\right) A x_{n}-y_{n}\right\|^{2}\right. \\
&\left.+\left\|x_{n}-x\right\|^{2}+2 \gamma\left\langle x_{n}-x, A^{*}\left(J_{r_{n}}^{N}-I\right) A x_{n}\right\rangle+\gamma^{2}\left\|A^{*}\left(J_{r_{n}}^{N}-I\right) A x_{n}\right\|^{2}\right\} \\
& \leq \frac{1}{2}\left\{\left\|y_{n}-x\right\|^{2}-\left(\left\|x_{n}-y_{n}\right\|^{2}+2 \gamma\left\langle x_{n}-y_{n}, A^{*}\left(J_{r_{n}}^{N}-I\right) A x_{n}\right\rangle+\left\|\gamma A^{*}\left(J_{r_{n}}^{N}-I\right) A x_{n}\right\|^{2}\right)\right. \\
&\left.+\left\|x_{n}-x\right\|^{2}+2 \gamma\left(\left\langle J_{r_{n}}^{N} A x_{n}-A x,\left(J_{r_{n}}^{N}-I\right) A x_{n}\right\rangle-\left\|\left(J_{r_{n}}^{N}-I\right) A x_{n}\right\|^{2}\right)+\gamma^{2}\|A\|^{2}\left\|\left(J_{r_{n}}^{N}-I\right) A x_{n}\right\|^{2}\right\} \\
& \leq \frac{1}{2}\left\{\left\|x_{n}-x\right\|^{2}+\gamma\left(\gamma\|A\|^{2}-1\right)\left\|\left(J_{r_{n}}^{N}-I\right) A x_{n}\right\|^{2}+\left\|y_{n}-x\right\|^{2}-\left\|x_{n}-y_{n}\right\|^{2}\right. \\
&\left.\left.+2 \gamma\left\|A\left(x_{n}-y_{n}\right)\right\|\left\|\left(J_{r_{n}}^{N}-I\right) A x_{n}\right\|-\left\|\gamma_{n} A^{*}\left(J_{r_{n}}^{N}-I\right) A x_{n}\right\|^{2}\right)\right\} \\
& \leq \frac{1}{2}\left\{\left\|y_{n}-x\right\|^{2}+\left\|x_{n}-x\right\|^{2}+2 \gamma\|A\|\left\|x_{n}-y_{n}\right\|\left\|\left(J_{r_{n}}^{N}-I\right) A x_{n}\right\|-\left\|x_{n}-y_{n}\right\|^{2}\right\} .
\end{aligned}
$$


It follows that

$$
\left\|y_{n}-x\right\|^{2} \leq\left\|x_{n}-x\right\|^{2}+2 \gamma\|A\|\left\|x_{n}-y_{n}\right\|\left\|\left(J_{r_{n}}^{N}-I\right) A x_{n}\right\|-\left\|x_{n}-y_{n}\right\|^{2} .
$$

This implies that

$$
\begin{aligned}
\left\|x_{n+1}-x\right\|^{2} & =\left\|T_{\beta_{n}} y_{n}-x-\mu \alpha_{n} F T_{\beta_{n}} y_{n}\right\|^{2} \\
& \leq\left\|T_{\beta_{n}} y_{n}-x\right\|^{2}-2 \mu \alpha_{n}\left\langle F T_{\beta_{n}} y_{n}, x_{n+1}-x\right\rangle \\
& \leq\left\|y_{n}-x\right\|^{2}+2 \mu \alpha_{n}\left\|F T_{\beta_{n}} y_{n}\right\|\left\|x_{n+1}-x\right\| \\
& \leq\left\|x_{n}-x\right\|^{2}+2 \gamma\|A\|\left\|x_{n}-y_{n}\right\|\left\|\left(J_{r_{n}}^{N}-I\right) A x_{n}\right\|-\left\|x_{n}-y_{n}\right\|^{2}+2 \mu \alpha_{n}\left\|F T_{\beta_{n}} y_{n}\right\|\left\|x_{n+1}-x\right\| .
\end{aligned}
$$

Thus,

$$
\begin{aligned}
\left\|x_{n}-y_{n}\right\|^{2} \leq & \left\|x_{n}-x\right\|^{2}-\left\|x_{n+1}-x\right\|^{2}+2 \gamma\|A\|\left\|x_{n}-y_{n}\right\|\left\|\left(J_{r_{n}}^{N}-I\right) A x_{n}\right\|+2 \mu \alpha_{n}\left\|F T_{\beta_{n}} y_{n}\right\|\left\|x_{n+1}-x\right\| \\
\leq & \left(\left\|x_{n}-x\right\|+\left\|x_{n+1}-x\right\|\right)\left\|x_{n}-x_{n+1}\right\|+2 \gamma\|A\|\left\|x_{n}-y_{n}\right\|\left\|\left(J_{r_{n}}^{N}-I\right) A x_{n}\right\| \\
& +2 \mu \alpha_{n}\left\|F T_{\beta_{n}} y_{n}\right\|\left\|x_{n+1}-x\right\| .
\end{aligned}
$$

From Lemmas 2.1 and 2.4, we find from (3.2) that

$$
\begin{aligned}
\left\|x_{n+1}-x\right\|^{2}= & \left\|\left(I-\mu \alpha_{n} F\right) T_{\beta_{n}} y_{n}-\left(I-\mu \alpha_{n} F\right) x\right\|^{2}+\mu^{2} \alpha_{n}^{2}\|F x\|^{2} \\
& -2 \mu \alpha_{n}\left\langle\left(I-\mu \alpha_{n} F\right) T_{\beta_{n}} y_{n}-\left(I-\mu \alpha_{n} F\right) x, F x\right\rangle \\
= & \left\|\left(I-\mu \alpha_{n} F\right) T_{\beta_{n}} y_{n}-\left(I-\mu \alpha_{n} F\right) x\right\|^{2}-\mu^{2} \alpha_{n}^{2}\|F x\|^{2} \\
& -2 \mu \alpha_{n}\left\langle\left(I-\mu \alpha_{n} F\right) T_{\beta_{n}} y_{n}-x, F x\right\rangle \\
\leq & \left(1-\tau \alpha_{n}\right)^{2}\left\|T_{\beta_{n}} y_{n}-T_{\beta_{n}} x\right\|^{2}-2 \mu \alpha_{n}\left\langle\left(I-\mu \alpha_{n} F\right) T_{\beta_{n}} y_{n}-x, F x\right\rangle \\
\leq & \left(1-\tau \alpha_{n}\right)^{2}\left\|y_{n}-x\right\|^{2}-2 \mu \alpha_{n}\left\langle\left(I-\mu \alpha_{n} F\right) T_{\beta_{n}} y_{n}-x, F x\right\rangle \\
\leq & \left(1-\tau \alpha_{n}\right)^{2}\left(\left\|x_{n}-x\right\|^{2}-\gamma_{n}\left(1-\gamma\|A\|^{2}\right)\left\|\left(J_{r_{n}}^{N}-I\right) A x_{n}\right\|^{2}\right) \\
& -2 \mu \alpha_{n}\left\langle x_{n+1}-x, F x\right\rangle \\
\leq & \left\|x_{n}-x\right\|^{2}-2 \mu \alpha_{n}\left\|x_{n+1}-x\right\|\|F x\|-\left(1-\tau \alpha_{n}\right)^{2} \gamma\left(1-\gamma\|A\|^{2}\right)\left\|\left(J_{r_{n}}^{N}-I\right) A x_{n}\right\|^{2} .
\end{aligned}
$$

It follows that

$$
\begin{aligned}
& \gamma\left(1-\gamma\|A\|^{2}\right)\left(1-\tau \alpha_{n}\right)^{2}\left\|\left(J_{r_{n}}^{N}-I\right) A x_{n}\right\|^{2} \\
& \leq\left\|x_{n}-x\right\|^{2}-\left\|x_{n+1}-x\right\|^{2}+2 \mu \alpha_{n}\left\|x_{n+1}-x\right\|\|F x\| \\
& \leq\left\|x_{n}-x_{n+1}\right\|\left(\left\|x_{n}-x\right\|+\left\|x_{n+1}+x\right\|\right)+2 \mu \alpha_{n}\left\|x_{n+1}-x\right\|\|F x\| .
\end{aligned}
$$

Hence,

$$
\lim _{n \rightarrow \infty}\left\|J_{r_{n}}^{N} A x_{n}-A x_{n}\right\|=0
$$

Using (3.12) and (3.13), one arrives at

$$
\lim _{n \rightarrow \infty}\left\|x_{n}-y_{n}\right\|=0
$$

Since $T_{\beta_{n}} y_{n}-y_{n}=\left(1-\beta_{n}\right)\left(T y_{n}-y_{n}\right)$, one obtains

$$
\lim _{n \rightarrow \infty}\left\|T y_{n}-y_{n}\right\|=0
$$


Since $F$ is strongly monotone and Lipschitz continuous, we next use $\bar{x}$ to denote the unique solution of variational inequality (3.1). Note that

$$
\left\langle F \bar{x}, \bar{x}-x_{n+1}\right\rangle=\left\langle F \bar{x}, \bar{x}-x_{n}\right\rangle+\left\langle F \bar{x}, x_{n}-x_{n+1}\right\rangle .
$$

Since $\left\{x_{n}\right\}$ is a bounded sequence, there exists a subsequence $\left\{x_{n_{k}}\right\}$ of $\left\{x_{n}\right\}$ such that $x_{n_{k}} \rightarrow x^{*}$ as $k \rightarrow \infty$.

Next, we show that $x^{*}$ is the common solution. From (3.15), we see from Lemma 2.5 that $x^{*}$ is a fixed point of $T$. On the other hand, one has

$$
\frac{x_{n}-y_{n}+\gamma A^{*}\left(J_{r_{n}}^{N} A x_{n}-A x_{n}\right)}{\gamma} \in M y_{n}
$$

Note that $y_{n_{k}} \rightarrow x^{*}$ as $k \rightarrow \infty$. It follows from (3.13) and (3.14) that $0 \in B x^{*}$. Since $A$ is a linear bounded operator, we find that $A x_{n_{k}} \rightarrow A x^{*}$ as $k \rightarrow \infty$. Using Lemma 2.2, we find that

$$
\begin{aligned}
\left\|J_{r_{n}}^{N} A x_{n}-J_{r}^{N} A x_{n}\right\| & =\left\|J_{r}^{N}\left(\frac{r}{r_{n}} A x_{n}+\left(1-\frac{r}{r_{n}}\right) J_{r_{n}}^{N} A x_{n}\right)-J_{r}^{N} A x_{n}\right\| \\
& \leq\left\|\frac{r}{r_{n}} A x_{n}+\left(1-\frac{r}{r_{n}}\right) J_{r_{n}}^{N} A x_{n}-A x_{n}\right\| \\
& \leq \frac{\left|r_{n}-r\right|}{r}\left\|J_{r_{n}}^{N} A x_{n}-A x_{n}\right\|,
\end{aligned}
$$

where $r$ is some positive real number. From (3.13), one obtains that $\left\|J_{r_{n}}^{N} A x_{n}-J_{r}^{N} A x_{n}\right\| \rightarrow \infty$ as $n \rightarrow \infty$. In view of

$$
\left\|J_{r}^{N} A x_{n}-A x_{n}\right\| \leq\left\|J_{r}^{N} A x_{n}-J_{r_{n}}^{N} A x_{n}\right\|+\left\|J_{r_{n}}^{N} A x_{n}-A x_{n}\right\|,
$$

we find that $\left\|J_{r}^{N} A x_{n}-A x_{n}\right\| \rightarrow \infty$ as $n \rightarrow \infty$. Since $J_{r}^{N}$ is nonexpansive, we find that $A x^{*} \in F i x\left(J_{r}^{N}\right)$, that is, $0 \in N A x^{*}$. This proves that $x \in \operatorname{SFP}(M, N) \cap \operatorname{Fix}(T)$. This shows from (3.16) that

$$
\limsup _{n \rightarrow \infty}\left\langle F \bar{x}, \bar{x}-x_{n+1}\right\rangle \leq 0 .
$$

Finally, we show that $\left\{x_{n}\right\}$ converges strongly to $\bar{x}$.

$$
\begin{aligned}
\left\|x_{n+1}-\bar{x}\right\|^{2} & =\left\|\left(I-\mu \alpha_{n} F\right) T_{\beta_{n}} y_{n}-\left(I-\mu \alpha_{n} F\right) \bar{x}-\mu \alpha_{n} F \bar{x}\right\|^{2} \\
& \leq\left\|\left(I-\mu \alpha_{n} F\right) T_{\beta_{n}} y_{n}-\left(I-\mu \alpha_{n} F\right) \bar{x}\right\|^{2}-2 \mu \alpha_{n}\left\langle F \bar{x}, x_{n+1}-\bar{x}\right\rangle \\
& \leq\left(1-\tau \alpha_{n}\right)^{2}\left\|T_{\beta_{n}} y_{n}-\bar{x}\right\|^{2}-2 \mu \alpha_{n}\left\langle F \bar{x}, x_{n+1}-\bar{x}\right\rangle \\
& \leq\left(1-\tau \alpha_{n}\right)^{2}\left\|y_{n}-\bar{x}\right\|^{2}-2 \mu \alpha_{n}\left\langle F \bar{x}, x_{n+1}-\bar{x}\right\rangle \\
& \leq\left(1-\tau \alpha_{n}\right)^{2}\left\|x_{n}-\bar{x}\right\|^{2}-2 \mu \alpha_{n}\left\langle F \bar{x}, x_{n+1}-\bar{x}\right\rangle \\
& \leq\left(1-2 \tau \alpha_{n}\right)\left\|x_{n}-\bar{x}\right\|^{2}+2 \alpha_{n} \mu\left\langle F \bar{x}, \bar{x}-x_{n+1}\right\rangle+\tau^{2} \alpha_{n}^{2}\left\|x_{n}-\bar{x}\right\|^{2} .
\end{aligned}
$$

Using Lemma 2.3, we get that $\left\|x_{n}-\bar{x}\right\| \rightarrow 0$. This completes the proof.

From Theorem 3.1, the following results are not hard to derived easily.

Corollary 3.2. Let $C$ be a nonempty closed and convex subset of a Hilbert space $H_{1}$ and let $Q$ be a nonempty closed and convex subset of a real Hilbert space $H_{2}$. Let $T$ be a nonexpansive mapping on $H_{1}$ with fixed points. Let $A: H_{1} \rightarrow H_{2}$ be a bounded linear operator and let $A^{*}$ be the adjoint operator of $A$. Let $F: H_{1} \rightarrow H_{1}$ be a $\mathscr{L}$-Lipschitz continuous and $\tau$-strongly monotone mapping. Let $M$ be a maximal monotone mapping on $H_{1}$ and let $\mathrm{N}$ be a maximal monotone mapping on $\mathrm{H}_{2}$. Assume that 
$\operatorname{Fix}(T) \cap \operatorname{SFP}(M, N) \neq \emptyset$. Let $\gamma$ be a positive real number. Let $\left\{\alpha_{n}\right\}$ be a sequence in $(0,1)$ and let $\left\{r_{n}\right\}$ be a positive sequence. Let $\left\{x_{n}\right\}$ be a sequence generated by the following process: $x_{1} \in H_{1}$ and

$$
\left\{\begin{array}{l}
x_{n+1}=T y_{n}-\mu \alpha_{n} F T y_{n}, \\
y_{n}=J_{r_{n}}^{M}\left(x_{n}+\gamma A^{*}\left(J_{r_{n}}^{N}-I\right) A x_{n}\right),
\end{array}\right.
$$

Assume that $\lim _{n \rightarrow \infty} \alpha_{n}=0, \sum_{n=1}^{\infty} \alpha_{n}=\infty$ and $\sum_{n=1}^{\infty}\left|\alpha_{n+1}-\alpha_{n}\right|<\infty, \gamma \in\left(0, \frac{1}{\|A\|^{2}}\right), \mu$ is a real number in $\left(0, \frac{2 \tau}{\mathscr{L}^{2}}\right),\left\{r_{n}\right\}$ is a real number sequence such that $\liminf _{n \rightarrow \infty} r_{n}>0, \sum_{n=1}^{\infty}\left|r_{n+1}-r_{n}\right|<\infty$. Then $\left\{x_{n}\right\}$ converge strongly to $\bar{x} \in \operatorname{SFP}(M, N) \cap F i x(T)$, which is the unique solution of the following variational inequality

$$
\langle F \bar{x}, \bar{x}-y\rangle \leq 0, \quad \forall y \in \operatorname{SFP}(M, N) \cap F i x(T) .
$$

Corollary 3.3. Let $C$ be a nonempty closed and convex subset of a Hilbert space $H_{1}$ and let $Q$ be a nonempty closed and convex subset of a real Hilbert space $H_{2}$. Let $A: H_{1} \rightarrow H_{2}$ be a bounded linear operator and let $A^{*}$ be the adjoint operator of $A$. Let $F: H_{1} \rightarrow H_{1}$ be a $\mathscr{L}$-Lipschitz continuous and $\tau$-strongly monotone mapping. Let $M$ be a maximal monotone mapping on $H_{1}$ and let $N$ be a maximal monotone mapping on $\mathrm{H}_{2}$. Assume that $\operatorname{SFP}(M, N) \neq \emptyset$. Let $\gamma$ be a positive real number. Let $\left\{\alpha_{n}\right\}$ be a sequence in $(0,1)$ and let $\left\{r_{n}\right\}$ be a positive sequence. Let $\left\{x_{n}\right\}$ be a sequence generated by the following process: $x_{1} \in H_{1}, x_{n+1}=y_{n}-\mu \alpha_{n} F y_{n}$, where $y_{n}=J_{r_{n}}^{M}\left(x_{n}+\gamma A^{*}\left(J_{r_{n}}^{N}-I\right) A x_{n}\right)$. Assume that $\lim _{n \rightarrow \infty} \alpha_{n}=0, \sum_{n=1}^{\infty} \alpha_{n}=\infty$ and $\sum_{n=1}^{\infty}\left|\alpha_{n+1}-\alpha_{n}\right|<\infty, \gamma \in\left(0, \frac{1}{\|A\|^{2}}\right), \mu$ is a real number in $\left(0, \frac{2 \tau}{\mathscr{L}^{2}}\right),\left\{r_{n}\right\}$ is a real number sequence such that $\liminf _{n \rightarrow \infty} r_{n}>0, \sum_{n=1}^{\infty}\left|r_{n+1}-r_{n}\right|<\infty$. Then $\left\{x_{n}\right\}$ converge strongly to $\bar{x} \in \operatorname{SFP}(M, N)$, which is the unique solution of the following variational inequality $\langle F \bar{x}, \bar{x}-y\rangle \leq 0$, $\forall y \in \operatorname{SFP}(M, N)$.

\section{Acknowledgments}

The authors are grateful to the referee for useful suggestions which improved this paper. The second author was supported by the Natural Science Foundation of Shandong Province of China (ZR2017LA001) and Youth Foundation of Linyi University (LYDX2016BS023).

\section{REFERENCES}

[1] Y. Censor, T. Elfving, A multiprojection algorithm using Bregman projections in a product space, Numer. Algorithms 8 (1994), 221-239.

[2] B.A. Bin Dehaish, et al., A regularization projection algorithm for various problems with nonlinear mappings in Hilbert spaces, J. Inequal. Appl. 2015 (2015), Article ID 51.

[3] S.Y. Cho, X. Qin, L. Wang, Strong convergence of a splitting algorithm for treating monotone operators, Fixed Point Theory Appl. 2014 (2014), Article ID 94.

[4] S.Y. Cho, B.A. Bin Dehaish, X. Qin, Weak convergence of a splitting algorithm in Hilbert spaces, J. Appl. Anal. Comput. 7 (2017), 427-438.

[5] S.Y. Cho, Generalized mixed equilibrium and fixed point problems in a Banach space, J. Nonlinear Sci. Appl. 9 (2016), 1083-1092.

[6] C. Byrne, Y. Censor, A. Gibali, S. Reich, Weak and strong convergence of algorithms for the split common null point problem, J. Nonlinear Convex Anal. 13 (2012), 759-775.

[7] Y. Censor, A. Gibali, S. Reich, Algorithms for the split varational inequality problem, Numer. Algorithms 59 (2012), 301-323.

[8] A. Moudafi, Split monotone variational inclusions, J. Optim. Theory Appl. 150 (2011), 275-283. 
[9] X. Qin, S.Y. Cho, L. Wang, Strong convergence of an iterative algorithm involving nonlinear mappings of nonexpansive and accretive type, Optimization, 67 (2018), 1377-1388.

[10] X. Qin, S.Y. Cho, Convergence analysis of a monotone projection algorithm in reflexive Banach spaces, Acta Math. Sci. 37 (2017), 488-502.

[11] N. Fang, Some results on split variational inclusion and fixed point problems in Hilbert spaces, Commun. Optim. Theory 2017 (2017), Article ID 5.

[12] N. Fang, Y. Gong, Viscosity iterative methods for split variational inclusion problems and fixed point problems of a nonexpansive mapping, Commun. Optim. Theory 2016 (2016), Article ID 11.

[13] L. Tian, L. Shi, R. Chen, Strong convergence theorems for split inclusion problems in Hilbert spaces, J. Fixed Point Theory Appl. 19 (2017), 1501-1514.

[14] J.Y.B. Cruz, Shehu, An iterative method for split inclusion problems without prior knowledge of operator norms, J. Fixed Point Theory Appl. 19 (2017), 2017-2036.

[15] X. Qin, J.C. Yao, Projection splitting algorithms for nonself operators, J. Nonlinear Convex Anal. 18 (2017), 925-935.

[16] F.E. Browder, W.V. Petryshyn, Construction of fixed points of nonlinear mappings in Hilbert spaces, J. Math. Anal. Appl. 20 (1967) 197-228.

[17] S.Y. Cho, X. Qin, On the strong convergence of an iterative process for asymptotically strict pseudocontractions and equilibrium problems, Appl. Math. Comput. 235 (2014), 430-438.

[18] H. Zhou, Convergence theorems of common fixed points for a finite family of Lipschitz pseudocontractions in Banach spaces, Nonlinear Anal. 68 (2008) 2977-2983.

[19] H. Zhou, Convergence theorems of fixed points for $\kappa$-strict pseudo-contractions in Hilbert spaces, Nonlinear Anal. 69 (2008), 456-462.

[20] H. Zhou, Convergence theorems for $\lambda$-strict pseudo-contractions in 2-uniformly smooth Banach spaces, Nonlinear Anal. 69 (2008), 3160-3173.

[21] X. Qin, S.Y. Cho, L. Wang, Iterative algorithms with errors for zero points of m-accretive operators, Fixed Point Theory Appl. 2013 (2013), Article ID 148.

[22] V. Barbu, Nonlinear Semigroups and Differential Equations in Banach Space, Noordhoff, Amsterdam, 1976.

[23] Z. Xue, H. Zhou, Y.J. Cho, Iterative solutions of nonlinear equations for m-accretive operators in Banach spaces, J. Nonlinear Convex Anal. 1 (2000), 313-320.

[24] Y. Yamada, The hybrid steepest-descent method for variational inequalities problems over the intersection of the fixed point sets of nonexpansive mappings, In: Butnariu, D., Censor, Y., Reich, S. (eds.) Inherently Parallel Algorithms in Feasibility and Optimization and Their Applications, pp. 473-504, North-Holland, Amsterdam, 2001. 